\title{
BRAIN EXTRACT INDUCES SYNAPTIC CHARACTERISTICS IN THE BASAL LAMINA OF CULTURED MYOTUBES ${ }^{1}$
}

\author{
JOSHUA R. SANES, ${ }^{2}$ DANIEL H. FELDMAN, JEANETTE M. CHENEY, AND \\ JOHN C. LAWRENCE, JR.* \\ Department of Physiology and Biophysics and ${ }^{*}$ Department of Pharmacology, Washington University School of Medicine, \\ St. Louis, Missouri 63110
}

Received May 19, 1983; Revised August 22, 1983; Accepted August 22, 1983

\begin{abstract}
The basal lamina (BL) that occupies the synaptic cleft of the skeletal neuromuscular junction is antigenically distinct from extrasynaptic muscle fiber $\mathrm{BL}$, rich in acetylcholinesterase (AChE), and bears projections that form junctional folds in the postsynaptic membrane. We report here that these synapse-specific features of BL are all present at low levels in embryonic rat myotubes cultured without nerve, and that their levels are markedly increased by addition of a soluble extract from adult rat brain. Light and electron microscopic methods show that: (1) antibodies which bind preferentially to synaptic BL in vivo stain small, discrete patches of the myotube's BL; (2) AChE accumulates in patches on the myotube surface; and (3) myotube BL and membrane form invaginations that resemble junctional folds. Patches of BL that bear synaptic antigens, AChE, or folds usually overlie clusters of acetylcholine receptors in the plasma membrane. Myotubes treated with a brain extract bear 5 to 20 times more junctional folds and patches rich in acetylcholine receptors, synaptic BL antigens, and AChE than control myotubes. Together with a previous demonstration that electrical and/or contractile activity can modulate the amount and composition of myotube BL (Sanes, J. R., and J. C. Lawrence, Jr. (1983) Dev. Biol. 97: 123-136), these results suggest that nerves could regulate differentiation of muscle fiber BL by a combination of activity-dependent and -independent mechanisms.
\end{abstract}

The basal lamina (BL) that ensheaths each skeletal muscle fiber lines the synaptic cleft of the neuromuscular junction. Although synaptic and extrasynaptic portions of this BL are similar in their fine structure (e.g., Zacks et al., 1973), and composition (Sanes, 1982), they differ in several ways. (1) Immunohistochemical studies have shown that in addition to antigens shared by synaptic and extrasynaptic areas, the BL contains components that are concentrated in either synaptic or extrasynaptic regions (Sanes and Hall, 1979; Sanes, 1982). (2) Acetylcholinesterase $(\mathrm{AChE})$, which hydrolyzes acetylcholine to terminate transmitter action, is concentrated in the

${ }^{1}$ We thank A. Chiu for supplying monoclonal antibodies, A. Trevor and A. E. Chung for gifts of antisera, V. Argiro for writing the morphometry program, and A. Chiu, J. Covault, J. Lichtman, and D. Purves for helpful comments. This work was supported by grants from the Muscular Dystrophy Association, the National Institutes of Health, and the National Science Foundation. J. S. is a Sloan Fellow and an Established Investigator of the American Heart Association. D. F. was a postdoctoral fellow of the Muscular Dystrophy Association. J. L. has received a Career Development Award from the Juvenile Diabetes Association. A preliminary report has appeared (Sanes et al., 1982).

2 To whom correspondence should be addressed. synaptic cleft; a large fraction of the synaptic enzyme is associated with BL (reviewed in Massoulie and Bon, 1982). (3) While extrasynaptic BL is planar, synaptic $\mathrm{BL}$ projects into junctional folds that invaginate the postsynaptic membrane. It is of interest to study the nature and origin of these synaptic specializations not only because they demonstrate distinct domains within one cell's BL, but also because synaptic BL is known to be important in the function and regeneration of the neuromuscular junction (reviewed in Sanes, 1983).

The precise alignment of synaptic BL with nerve terminals argues that nerve and muscle collaborate in dividing BL into distinct synaptic and extrasynaptic domains. Two ways in which nerve is thought to regulate the differentiation of muscle are by evoking electrical activity, through the conventional process of synaptic transmission, and by releasing soluble "trophic" agents (reviewed in Purves, 1976; Harris, 1980). A strategy for studying these mechanisms has been to culture myotubes without neurons, and then to assess the effects of modulating their activity or of supplementing their medium with neural extracts (Cohen and Fischbach, 1973; Oh, 1975; Christian et al., 1978; Podleski et al., 1978; Jessell 
et al., 1979; Rieger et al., 1980; Rubin et al., 1980; Strohman et al., 1981; Kalcheim et al., 1982a, b; Markelonis et al., 1982; Salpeter et al., 1982). We have used this approach to study the formation of myotube BL.

Cultured rat myotubes assemble a discontinuous BL. Several shared antigens are present throughout this BL, while synaptic antigens are concentrated in discrete patches that usually coincide with acetylcholine receptor (AChR)-rich areas (Silberstein et al., 1982; Sanes and Lawrence, 1983). By comparing spontaneously active to chronically paralyzed myotubes, we previously found that accumulation of BL by myotubes is activity-dependent: active myotubes bear more total BL but fewer synaptic antigen-rich patches than paralyzed myotubes (Sanes and Lawrence, 1983). We have now characterized the syna,tic antigen-rich patches of BL further and studied the effect of a soluble extract of rat brain on their formation. We show here that brain extract selectively induces the accumulation of BL that resembles synaptic $\mathrm{BL}$ in several respects. Thus, activity and soluble neural factors both affect accumulation of BL, but in different ways, suggesting that nerves could regulate the differentiation of muscle BL by a combination of activitydependent and -independent mechanisms.

\section{Materials and Methods}

Muscle cultures and brain extract. Muscle was cultured from 19- to 20-day embryonic rats as described previously (Lawerence and Catterall, 1981; Sanes and Lawrence, 1983). Briefly, cells were dissociated from forelimb muscle, plated on collagen-coated polystyrene coverslips, and grown in Dulbecco's modified Eagle's medium supplemented with $10 \%$ horse serum, $5 \%$ newborn calf serum, and antibiotics. Initially, all cells in the cultures were mononucleated; cells fused to form multinucleated myotubes from day 2 to day 5. Cultures were treated with cytosine arabinoside $\left(10^{-5} \mathrm{M}\right)$, from day 4 to day 8 , to limit growth of fibroblasts, and studied during the second week in culture.

Eight to 11 days after plating, some cultures were refed with medium containing $10 \mu \mathrm{M}$ tetrodotoxin (Calbiochem), $1 \mathrm{~mm}$ lidocaine (Sigma), and/or a soluble extract of adult rat brain. The extract was prepared from whole brains of 100- to 400-gm Sprague-Dawley rats. Brains were homogenized, first in a Teflon-glass tissue grinder and then in a Polytron (Brinkman Instruments), at a concentration of $25 \%(\mathrm{w} / \mathrm{v})$ in Dulbecco's saline containing $10 \%$ horse serum. The homogenate was centrifuged at $105,000 \times \mathrm{g}$ for $90 \mathrm{~min}$ or $27,000 \times \mathrm{g}$ for $4 \mathrm{hr}$, and the supernatant was sterilized by filtration, divided into aliquots, and stored at $-70^{\circ} \mathrm{C}$. In a few experiments, similar extracts were made from embryonic (day 19 to 20) brain, adult spinal cord, or adult liver. For use, extract was thawed, diluted $1 / 10$ with standard medium containing cytosine arabinoside, and added to the cultures. Control cultures were incubated with anesthetic and extract-free but otherwise identical medium. Except where noted, "extract-treated" cultures were studied 3 to 4 days after a single application of extract-that is, 11 to 15 days after plating.

Because rat brain is rich in soluble $\mathrm{AChE}$, we performed a control experiment in which the $\mathrm{AChE}$ in brain extract was irreversibly inhibited with the inhibitor, diisopropylfluorophosphate (DFP;Sigma), before the extract was added to the cultures. Extract and DFP (50 $\mu \mathrm{M}$ final concentration) were mixed and incubated for $5 \mathrm{hr}$ at $4^{\circ} \mathrm{C}$. This treatment inhibited $\mathrm{AChE}$ in the extract by $>95 \%$, as assayed by the method of Ellman et al. (1961). The extract was then diluted $1 / 10$ with medium and incubated $3 \mathrm{hr}$ more. Residual DFP was thereby rendered ineffective, as shown by the inability of an aliquot of the DFP-containing medium to inhibit AChE in an equal aliquot of fresh brain extract. Dilution, hydrolysis, and inactivation by serum proteins may all have contributed to the neutralization of free DFP (Koelle, 1963). Analogous experiments with $\mathrm{C} 1$ antigen (described below) proved infeasible. Although preincubation of $\mathrm{C} 1$ with brain extract did not affect the ability of the antibody to stain cultures subsequently, neither could we satisfactorily absorb $\mathrm{C} 1$ with cultured myotubes.

Histology. For immunofluorescence, cultures were incubated with antibody, washed, incubated with a mixture of fluorescein "second antibody" (anti-IgG) and rhodamine- $\alpha$-bungarotoxin, washed again, fixed in $2 \%$ paraformaldehyde, and mounted. The cultures were then viewed and photographed with filters that allowed detection of each fluorophore separately (see Sanes and Hall, 1979; Sanes and Lawrence, 1983 for details).

'To demonstrate sites of AChE activity, cultures were fixed in paraformaldehyde for $15 \mathrm{~min}$, washed, and stained for 1 to $2 \mathrm{hr}$ by the histochemical method of Karnovsky (1964). In some experiments, the inhibitors eserine, 1,5-bis-(4-allyldimethyl-ammoniumphenyl)pentan-3-one dibromide (=BW284C51), or tetraisopropyl pyrophosphoramide (=iso-OMPA) (all from Sigma) were added to the incubation mixture.

Cultures were fixed and stained for electron microscopy in one of four ways. (1) For conventional morphological study, cultures were fixed for $15 \mathrm{~min}$ in $1 \%$ paraformaldehyde, $1 \%$ glutaraldehyde, $110 \mathrm{mM} \mathrm{NaCl}, 5$ $\mathrm{mM} \mathrm{CaCl} 2,30 \mathrm{mM}$ HEPES, $\mathrm{pH}$ 7.2. They were then rinsed in $0.1 \mathrm{M}$ sodium cacodylate, $\mathrm{pH} 7.2$, refixed in $1 \%$ $\mathrm{OsO}_{4}$ in cacodylate, washed, dehydrated, and embedded in Polybed 812 (Polysciences). The coverslip was then peeled off, and thin sections cut perpendicular to the culture's surface were stained with uranyl acetate and lead citrate. (2) To demonstrate sites of AChE activity, cultures were stained histochemically (Karnovsky, 1964) after aldehyde and before $\mathrm{OsO}_{4}$ fixation. (3) For immunocytochemistry, cultures were incubated with antibodies as described for immunofluorescence, except that horseradish peroxidase (HRP)-conjugated second antibody was used instead of fluorescein-antibody. The cultures were then fixed in aldehydes, stained for HRP (Streit and Reubi, 1977), refixed in $\mathrm{OsO}_{4}$, dehydrated, embedded, and sectioned. (4) To demonstrate sites of high AChR density, cultures were incubated for $1 \mathrm{hr}$ with HRP- $\alpha$-bungarotoxin, prepared by the method of Lentz et al. (1977), and generously supplied by Ted Usdin (Washington University). Cultures were then washed, fixed, stained for HRP, and prepared as described above.

Antibodies. The serum and monoclonal antibodies we used all bind to the BL of adult muscle fibers. Previous publications have described the preparation of polyclonal 
antibodies and the way in which they stain adult muscle (Sanes and Hall, 1979; Sanes, 1982) and cultured myotubes (Sanes and Lawrence, 1983). Antibodies that bind selectively to synaptic BL were prepared by Sanes and Hall (1979) and are designated JS1, JS2, and JS3 for "junction-specific," as proposed by Silberstein et al. (1982). These antibodies bind to three different determinants in synaptic BL (Sanes and Hall, 1979). Antiserum to bovine brain AChE (Greenberg et al., 1977), which cross-reacts with rat muscle (Sanes and Hall, 1979), was provided by Drs. A. Trevor and A. Greenberg (University of California, San Francisco). Antiserum to GP-2 (Chung et al., 1979), a subunit of laminin (Sakashita et al., 1980), was the gift of Dr. A. E. Chung (University of Pittsburgh). Antisera to basement membrane collagen-rich fractions from muscle and lens capsule were prepared by Sanes and Hall (1979); bleeds were used that had little synapse-specific antibody (see Sanes and Lawrence, 1983).

Monoclonal antibodies that bind to muscle BL were prepared by Dr. Arlene Chiu (Sanes and Chiu, 1983). Antibodies $\mathrm{C} 1$ and $\mathrm{C} 4$ stain synaptic $\mathrm{BL}$ far more intensely than extrasynaptic BL in adult muscle, and thus recognize synaptic antigens, while $\mathrm{C} 10$ stains synaptic and extrasynaptic BL with comparable intensity, and thus recognizes a shared antigen. A connective tissuerich fraction from rat skeletal muscle was used as immunogen to produce $\mathrm{C} 1$ and $\mathrm{C} 10$, while $\mathrm{C} 4$ was raised against bovine lens capsule. All three monoclonal antibodies are IgG1.

Preimmune rabbit sera and a non-cross-reacting monoclonal antibody (IgG) to a chick antigen were used as controls. In neither immunofluorescence nor immunoperoxidase experiments was the myotube surface detectably stained when anti-BL was omitted or replaced with control antibody. However, debris and extracellular fibrils were nonspecifically stained; therefore, we confined our observations to myotube-associated immunoreactive material. All "second antibodies"-fluorescein- and HRP-conjugated goat anti-mouse and anti-rabbit IgGwere purchased from Cappel (Cochranville, PA).

\section{Results}

Myoblasts dissociated from embryonic rat muscle fused to form myotubes 2 to 5 days after plating. By 1 week in culture, many myotubes were striated and the majority twitched spontaneously. As the myotubes matured, they acquired a BL that was discontinuous but ultrastructurally similar to the continuous BL of adult muscle fibers (Fig. 1; see Sanes and Lawrence, 1983). We found that a few small patches of myotube BL resembled the synaptic BL of innervated muscle in several respects, and that treatment with a soluble brain extract selectively enhanced the expression of each of these synaptic features. The synaptic features we studied are synapsespecific BL antigens, structures that resemble junctional folds, and focal accumulations of AChE. We also monitored the formation of clusters of AChRs in the myotubes' plasma membrane.

Synaptic BL antigens. We have generated several polyclonal and monoclonal antibodies that stain synaptic BL far more intensely than extrasynaptic BL in adult muscle (Sanes and Hall, 1979; Sanes and Chiu, 1983). Each of these antibodies bound to small discrete patches on the surface of cultured myotubes (Fig. $2 a$; see also Sanes and Lawrence, 1983). Double staining with fluorescein-antibody and rhodamine- $\alpha$-bungarotoxin (which binds to AChRs) showed that patches rich in synaptic $\mathrm{BL}$ antigens usually coincided with regions of high $\mathrm{AChR}$

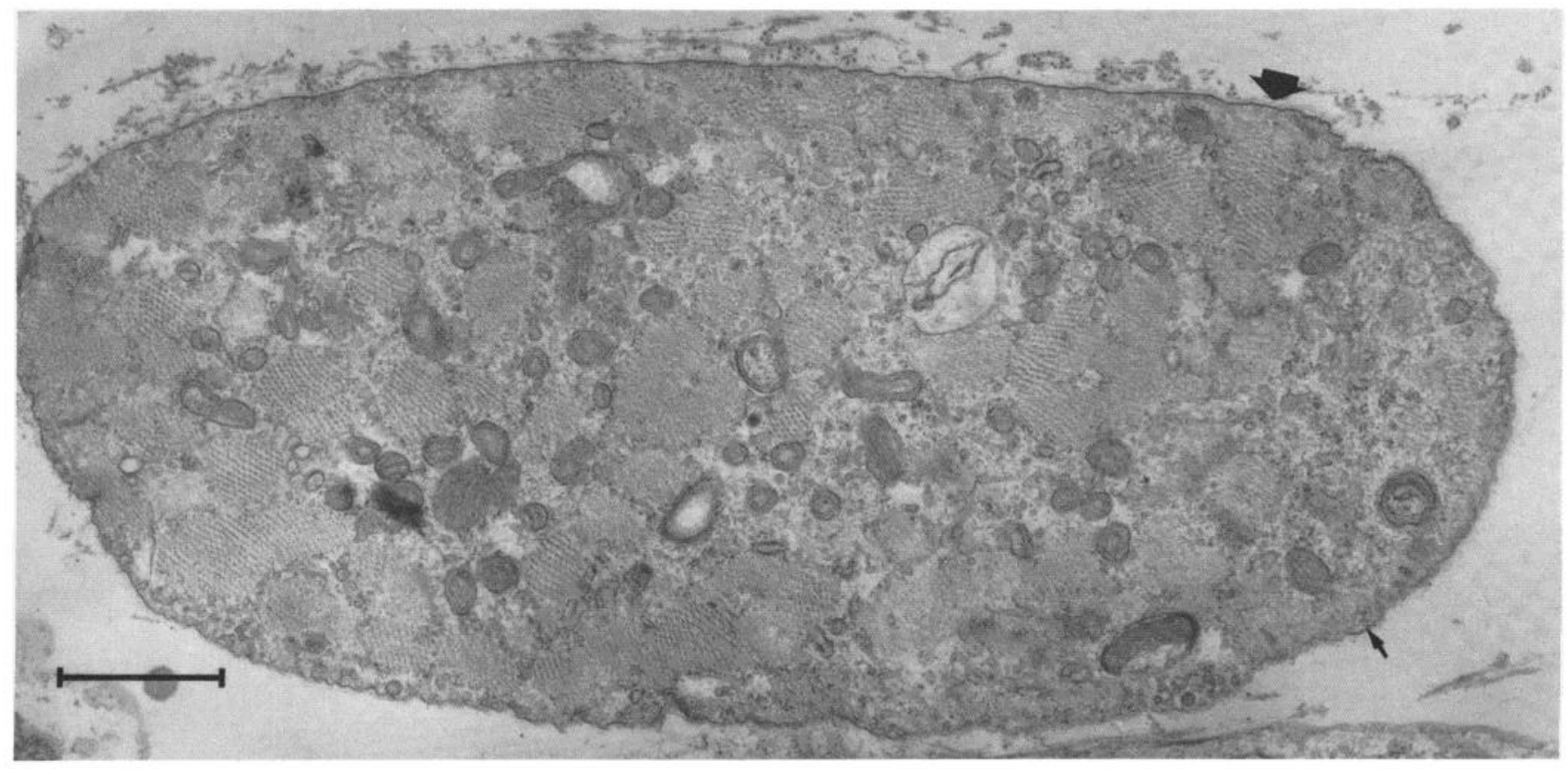

Figure 1. Cross-section of a cultured myotube. A BL coats some (large arrowhead) but not all (small arrowhead) of the cell's plasma membrane. Extracellular fibrils that resemble reticular lamina lie beyond the BL. Organized contractile elements fill the cytoplasm. The bar is $1 \mu \mathrm{m}$. 

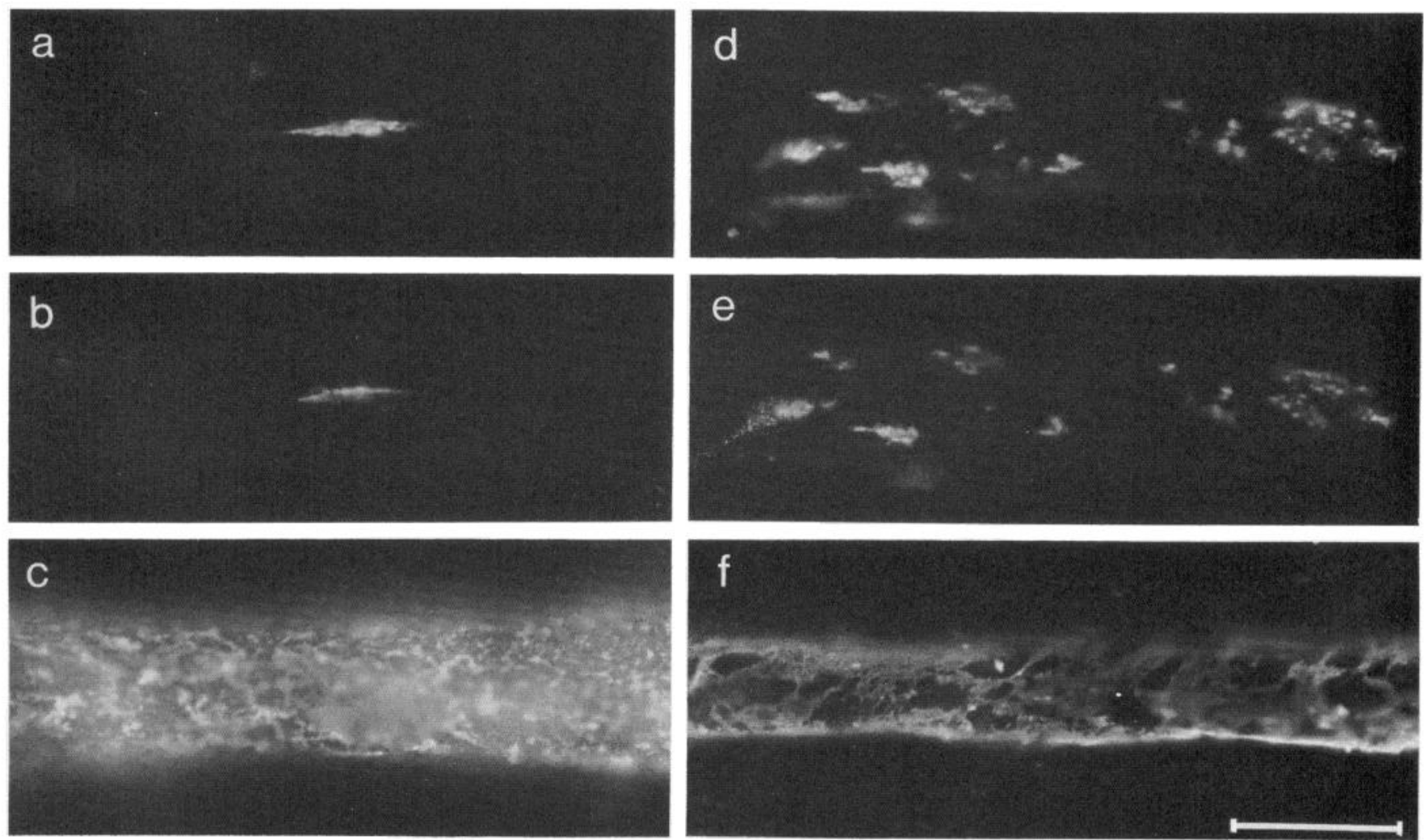

Figure 2. Antibodies to BL stain control ( $a$ to $c$ ) and brain extract-treated ( $d$ to $f$ ) myotubes. Cultures were incubated with antibody $\mathrm{C} 1$ (to a synaptic BL antigen) or anti-GP2 (a shared BL antigen), fluorescein-second antibody, and rhodaminebungarotoxin; then they were photographed with fluorescein or rhodamine optics. $a$ and $b$, Control myotube stained with $\mathrm{C} 1$ ( $a$, fluorescein) and bungarotoxin ( $b$, rhodamine); the antibody-stained patch is at an AChR-rich region. $c$, Control myotube stained with anti-GP2; much of the myotube surface is stained. $d$ and $e$, Extract-treated myotube stained with $\mathrm{C} 1$ ( $d$, fluorescein) and bungarotoxin (e, rhodamine). $f$, Extract-treated myotube stained with anti-GP2. The bar is $50 \mu \mathrm{m}$.

density (Fig. 2b). Electron microscopy of immunoperoxidase-stained cultures confirmed that synaptic antigens were concentrated in patches of BL (Fig. $3 a$ ).

Myotubes incubated with a crude soluble extract of adult rat brain accumulated severalfold more patches rich in synaptic BL antigens and in AChRs than control myotubes (Fig. 2, $d$ and $e$ ). Effects of extract on BL were seen with synapse-specific antibodies JS1, JS2, JS3, C1, and C4 (see "Materials and Methods"); monoclonal antibody $\mathrm{C} 1$ was used for most experiments. The number of C1-stained patches per myotube increased within 14 $\mathrm{hr}$ of adding extract to the cultures, and a 10 -fold effect was apparent after 1 day (Fig. 4). In extract-treated as in control cultures, and at all times tested after adding extract, the coincidence between $\mathrm{C} 1$ - and bungarotoxinstained patches was high: 80 to $90 \%$ of AChR-rich regions were stained by $\mathrm{C} 1$, and 90 to $95 \%$ of $\mathrm{C} 1$-stained patches were rich in AChRs. Extracts of embryonic brain or adult spinal cord had effects on BL similar to those of adult brain, whereas an identically prepared extract of adult liver had no detectable effect. Thus, neural extracts enhance synthesis or accumulation of synaptic antigen-rich BL.

Since accumulation of synaptic BL antigens is known to be activity-dependent (Sanes and Lawrence, 1983), it was important to ask whether brain extract affected BL by modulating the spontaneous electrical or contractile activity of the myotubes. Therefore, we measured the effect of extract on myotubes that were chronically paralyzed with the local anesthetic lidocaine or with the action potential blocker tetrodotoxin. Concentrations of lidocaine and tetrodotoxin used blocked all visible contractile activity. Paralysis alone increased the number of synaptic BL antigen- and AChR-rich patches, as previously observed, but extract caused a further increase in the accumulation of these patches (Fig. $5, a$ and $b$ ). Thus, while accumulation of synaptic antigen-rich BL is regulated by activity, the effects of extract on synaptic BL are largely activity-independent.

To determine whether the effect of brain extract on synaptic antigen-rich BL was selective, we compared the total fraction of the myotube surface covered by BL in control and extract-treated cultures, using both light and electron microscopy. For immunofluorescence, cultures were stained with antibodies such as anti-GP2 (=laminin) that stain the entire myotube BL in vitro (Sanes and Lawrence, 1983; see also Kuhl et al., 1982); these are antibodies to antigens shared by synaptic and extrasynaptic BL in vivo (Sanes, 1982). Brain extract had no large effect on the fraction of the myotube surface stained by antibodies to shared antigens (Fig. $2 c$ and $f$ ), although in some experiments extract-treated cells were somewhat more fully stained than controls. For electron microscopy, BL was stained either conventionally, with heavy 

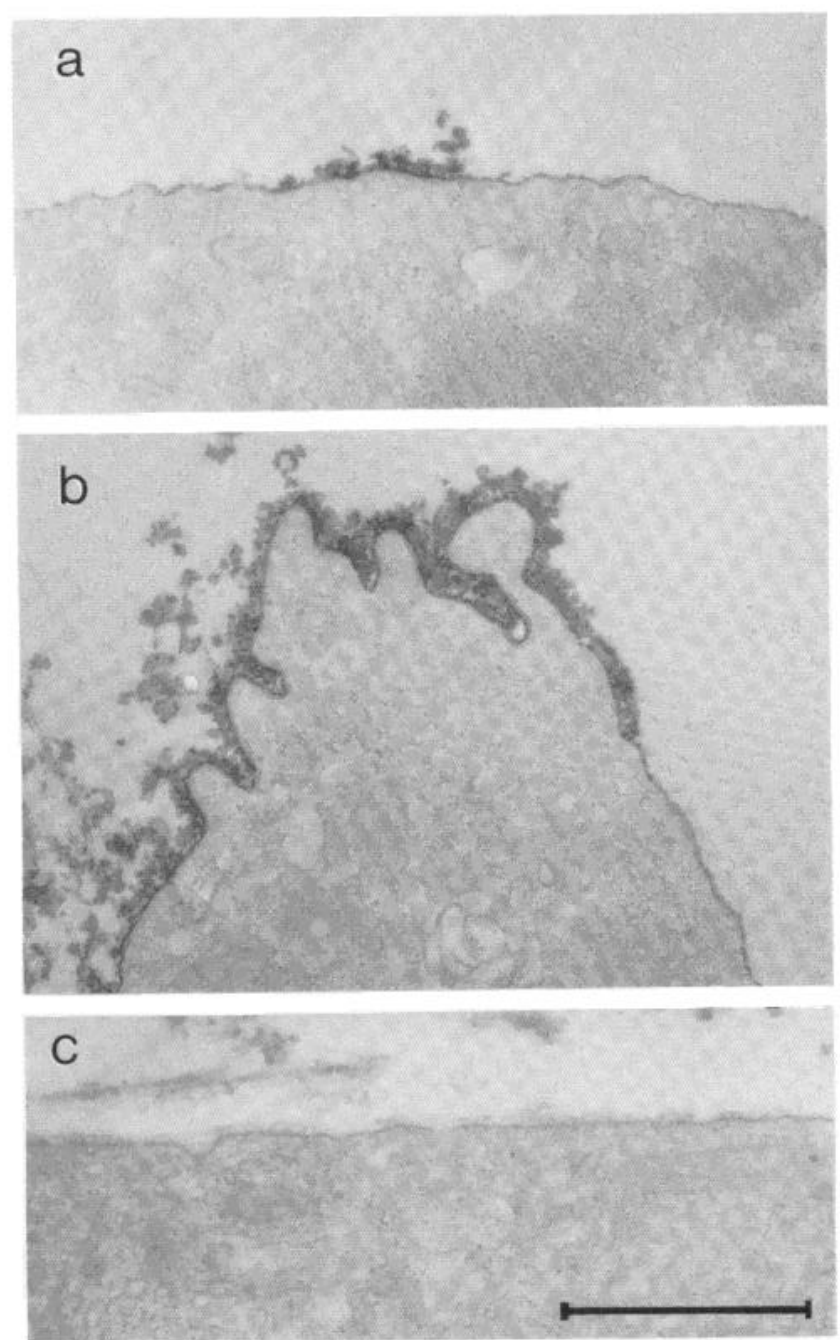

Figure 3. $a$ and $b$, Patches of synaptic antigen-rich BL, on the surface of control $(a)$ and brain extract-treated $(b)$ myotubes. Cultures were incubated with antibody $\mathrm{C} 1$ and HRPsecond antibody and then were fixed, stained for HRP, embedded, and sectioned for electron microscopy. $c$, Unstained $\mathrm{BL}$, from a culture incubated with irrelevant antibody instead of $\mathrm{C} 1$, but othewise treated identically to $a$ and $b$. The bar is 1 $\mu \mathrm{m}$.

metals, or immunocytochemically, with antibodies to shared BL antigens. Myotubes were cross-sectioned, and the fraction of their perimeters covered by stained $\mathrm{BL}$ was measured. Brain extract had little effect on the overall accumulation of myotube BL, while extract-induced increases in synaptic antigen-rich $\mathrm{BL}$, were readily detected in electron micrographs of myotubes stained with antibodies to synaptic BL (Fig. 6). It is possible that small differences between control and extracttreated cultures would have been missed by the immunoperoxidase method: peroxidase reaction product can diffuse before precipitating (Novikoff et al., 1972; J. M. Cheney and J. R. Sanes, unpublished observations), thus disproportionately increasing the apparent size of small antibody-binding areas. This artifact would minimize differences between control and extract-treated cultures and between values obtained with synaptic and shared antibodies. Despite this reservation, however, the electron microscopic measurements clearly support the conclusion that, at the concentration used, brain extract selectively increases accumulation of synaptic BL.

Junctional folds. In adult muscle, projections of BL extend from the synaptic cleft into the $\sim 1-\mu$ m-deep junctional folds that indent the postsynaptic membrane (Fig. 7a). These projections are absent from extrasynaptic regions and serve to mark synaptic sites in situ (Sanes et al., 1978). While studying the BL of immunoperoxidase-stained myotubes, we occasionally encountered structures that resembled junctional folds (Fig. $3 b$ ). Similar invaginations could be found in conventionally stained myotubes (Fig. $7 b$ ); thus, they were not induced by antibody binding. Folds were 0.3 to $1 \mu \mathrm{m}$ deep and about 0.15 to $0.3 \mu \mathrm{m}$ wide at their mouths. Views of single cross-sections showed that folds occur in small groups (range 1 to 4 , mean $=1.8$ ). Folds in the myotube surface almost invariably $(>95 \%)$ occurred in BL-covered regions and were coated with BL throughout their depth. The BL that lined the folds was continuous with that on the surface. Sometimes a pleat of BL extended from surface into fold, but more often BL within a fold intersected surface BL to form a "T." In all these respects, the folds resembled junctional folds seen in vivo. Systematic examination of many cultures showed that folds were far more common in brain extract-treated than in control or paralyzed myotubes (Table I). Thus, folds constitute another synapse-specific feature of the myotube surface and of its BL that is induced or stabilized by brain extract.

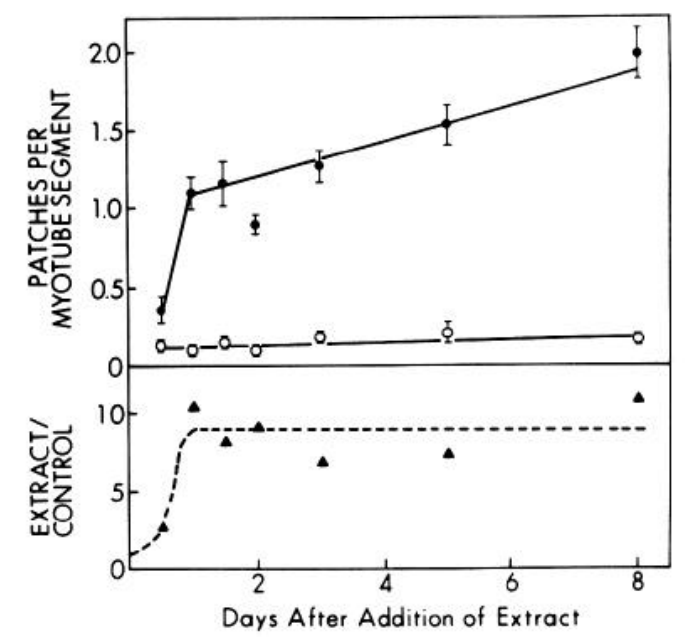

Figure 4. Brain extract induces formation of synaptic BLrich patches. At indicated times after addition of extract, cultures were stained with $\mathrm{C} 1$ and fluorescein-second antibody and viewed at $\times 400$. For each of 15 to 20 evenly spaced fields per culture, the number of myotubes that crossed the field and the number of fluorescein-stained patches per field were counted. Each point represents one culture. $a$, Average number of $\mathrm{C} 1$-stained patches per myotube segment that crossed the microscope field, $\pm \mathrm{SE}$, in extract-treated $(0)$ and matched control (O) cultures. Each point represents one culture. A second dose of extract added to cultures 4 days after the first had little additional effect (not shown). Control and extracttreated myotubes did not differ markedly in number or diameter. $b$, Ratio of C1-stained patches in extract-treated to control cultures, calculated from data in $a$. 


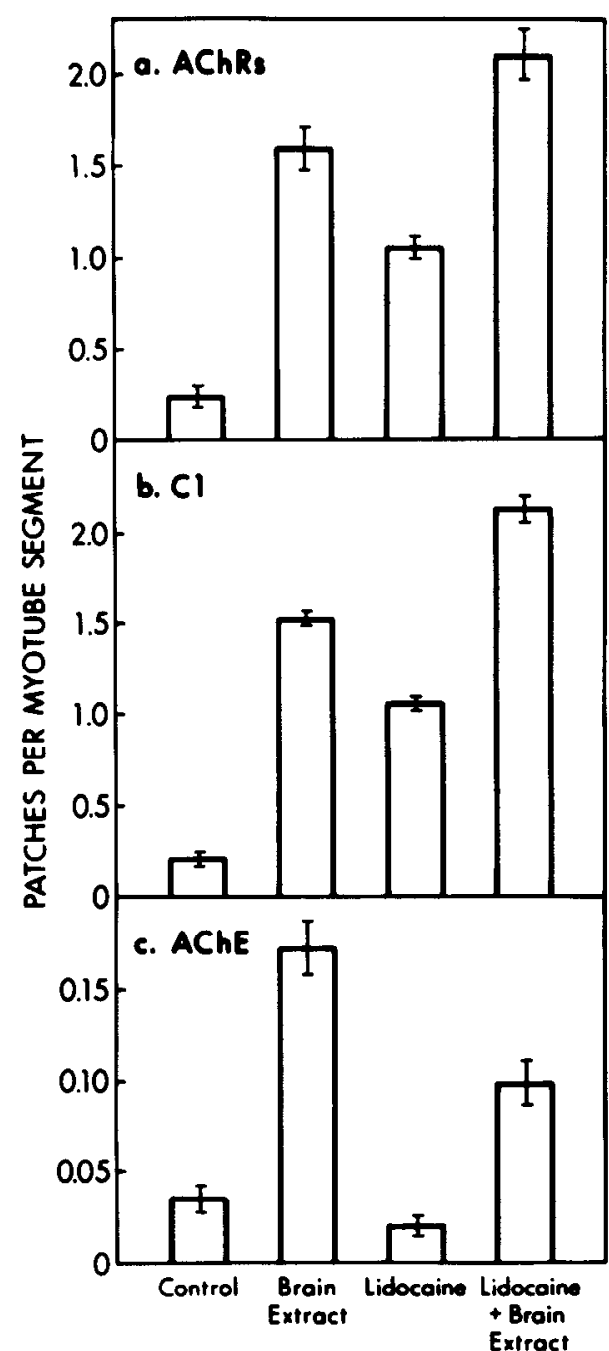

Figure 5. Brain extract induces synaptic differentiation of the myotube surface in active and paralyzed myotubes. Cultures were treated with the anesthetic lidocaine and/or brain extract from day 10 to day $15(a, b)$ or from day 8 to $11(c)$, then they were stained as described under "Materials and Methods" and scored as described in the legend to Figure 4. a, AChR-rich patches, stained with rhodamine-bungarotoxin. $b$, Synaptic BLrich patches, stained with $\mathrm{C} 1$ and fluorescein-second antibody. $c$, AChE-rich patches, stained histochemically. In $a$ and $b$, which are from the same experiment, each bar represents mean $\pm \mathrm{SE}$ of values from 20 fields in a single culture. In c, from a separate experiment, each bar represents mean $\pm \mathrm{SE}$ of values from 60 fields in three cultures. Similar results were obtained when tetrodotoxin instead of lidocaine was used to block activity.

Acetylcholinesterase. AChE is associated with synaptic $\mathrm{BL}$ at the adult neuromuscular junction (Massoulie and Bon, 1982). Since cultured rat myotubes are known to synthesize $\mathrm{AChE}$, including its synapse-associated $16 \mathrm{~S}$ form (Rieger et al., 1980; Brockman et al., 1982), we used a histochemical stain (Karnovsky, 1964) to determine whether brain extract could affect the distribution of surface-associated AChE in our cultures. We found very few patches of reaction product on the surface of histochemically stained control myotubes. However, severalfold more stained patches appeared on extract-treated myotubes (Fig. 8). In a survey of 10 control and 10 extract-treated cultures from six platings, we found an average of $3.2 \pm 0.6$ stained patches per $\times 40$ field in extract-treated cultures $(0.21$ patches per myotube segment, calculated as in Figs. 4 and 5), but only $0.49 \pm 0.05$ patches per field ( $=0.03$ per myotube segment) in control cultures. Thus, brain extract induced the formation of focal accumulations of cholinesterase.

Further histochemical and immunochemical studies showed that the reaction product we saw represented AChE that was made by the cultures and located on the myotube surface. Histochemical staining of cultured myotubes was very weak compared to that found at synaptic sites in vivo: patches were lightly stained even when the histochemical reaction was prolonged for $2 \mathrm{hr}$, whereas comparably fixed adult neuromuscular junctions stained intensely in about $5 \mathrm{~min}$. Nonetheless, reaction product was attributable to AChE, since staining of both control and extract-treated myotubes was abolished by the cholinesterase inhibitor eserine $\left(10^{-5} \mathrm{M}\right)$ and by the inhibitor of "true" AChE, BW 284c51 $\left(10^{-6} \mathrm{M}\right)$, but not by the inhibitor of nonspecific cholinesterase, iso-OMPA $\left(10^{-4} \mathrm{M}\right)$. Furthermore, occasional patches on extracttreated myotube were faintly but clearly stained by an antibody to AChE; these patches coincided with AChRrich areas. AChE accumulation occurred after addition of extract in which endogenous AChE had been irreversibly inhibited by DFP (see "Materials and Methods"); thus, extract induced rather than contributed the AChE in the patches. Electron microscopy of histochemically stained, extract-treated cultures (Fig. $8 b$ ) showed that

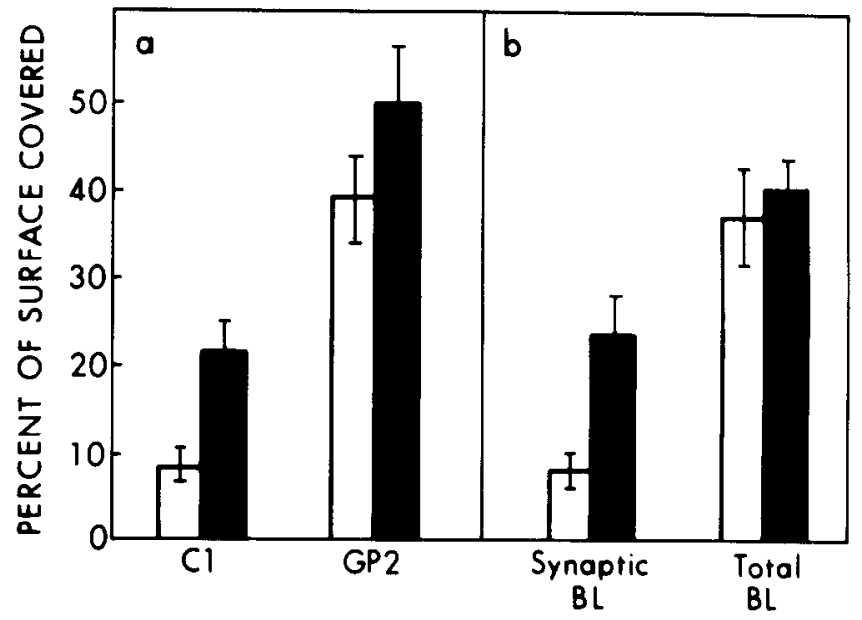

Figure 6. Brain extract selectively enhances accumulation of synaptic antigen-rich BL.Cultures were stained conventionally or by an immunoperoxidase method. Electron micrographs were prepared of cross-sectioned myotubes (final magnification, $\times 16,000$ ), and the percentage of the surface covered by stained BL was measured with a microcomputer-based planimetry system. Open bars represent control cultures, and solid bars represent extract-treated cultures. In $a$, each bar represents mean $\pm \mathrm{SE}$ of measurements from 15 to 25 randomly selected myotubes ( 5 to 10 from each of three separate blocks) in a single culture stained with $\mathrm{C} 1$ or anti-GP2. In $b$, each bar represents mean $\pm \mathrm{SE}$ of mean values from eight cultures. Synaptic $B L$ includes four experiments with JS1, two with $\mathrm{C} 1$, and one each with JS2 and JS3. Total $B L$ includes two experiments each with $\mathrm{C} 10$, anti-GP2, anti-muscle basement membrane collagen, and conventional staining. 

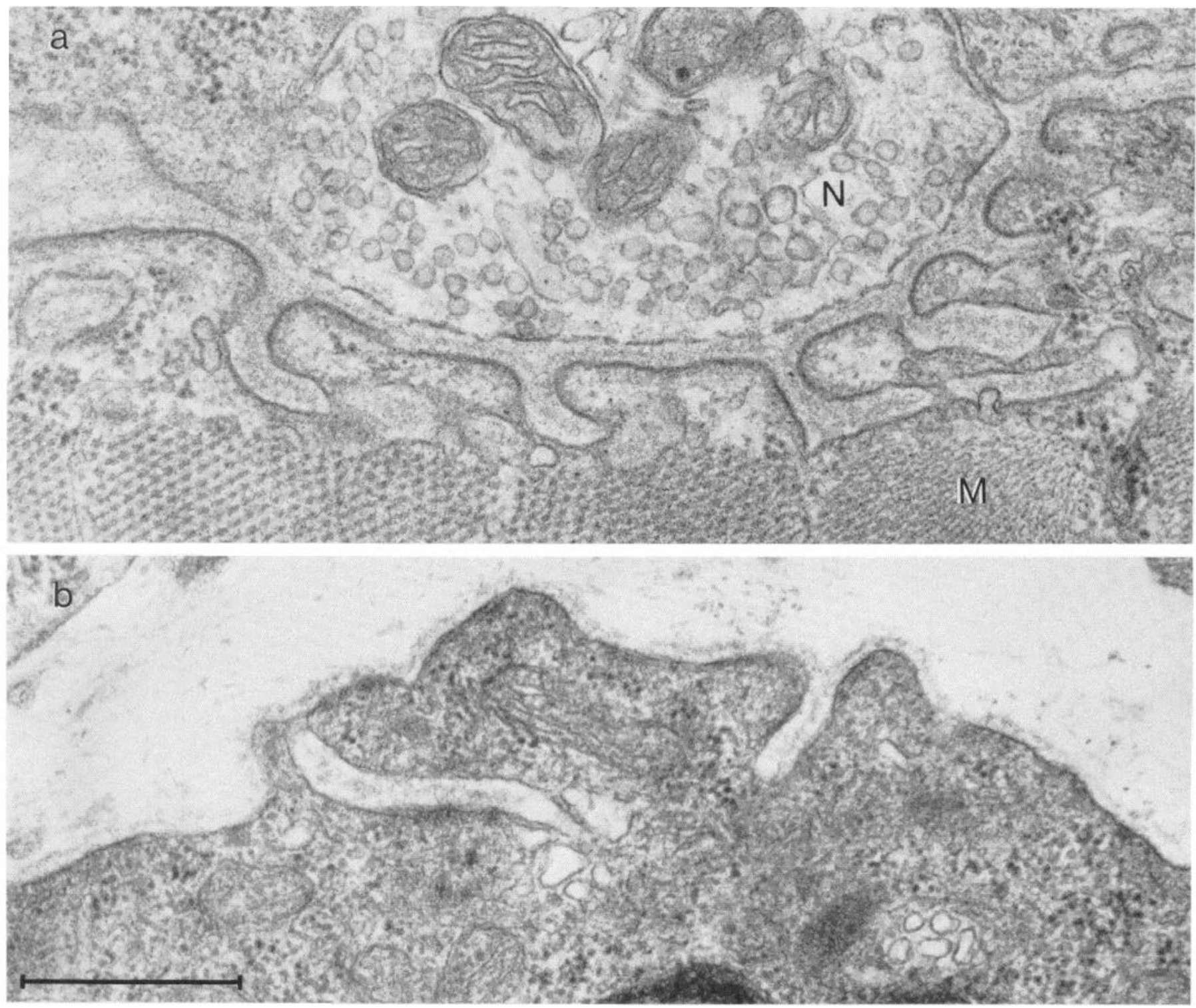

Figure 7. $a$, Portion of a neuromuscular junction from adult rat soleus muscle, to show junctional folds. $N$, nerve terminal; $M$, muscle fiber. $b$, Structures resembling junctional folds in an extract-treated cultured myotube. The bar is $0.5 \mu \mathrm{m}$.

deposits of reaction product were almost invariably on BL-coated areas of the myotube surface ( 36 of 38 patches found in three cultures), most of which ( 23 of $38=61 \%$ ) bore folds.

Brain extract induced focal accumulation of $\mathrm{AChE}$ in myotube paralyzed with TTX or lidocaine, as well as in spontaneously active myotubes (Fig. $5 c$ ). However, as expected from previous work showing that $\mathrm{AChE}$ accumulation is activity-dependent (Cangiano et al., 1980; Lømo and Slater, 1980; Rieger et al., 1980; Rubin et al., 1980), fewer AChE-rich patches were seen on paralyzed than on active myotubes in either the presence or absence of brain extract. Thus, while AChE and other synaptic BL antigens are oppositely affected by activity (compare Fig. 5, $b$ and $c$ ), they are regulated in parallel and in an activity-independent fashion by brain extract.

Coincidence of synaptic specialization. Our results show that brain extract enhances the formation of regions rich in $\mathrm{AChRs}$, synaptic BL antigens, $\mathrm{AChE}$, and structures resembling junctional folds. Although it has not been feasible to view all of these features in the same preparation, various experiments in which specializations were viewed two at a time demonstrate that the degree to which they coincide is high. (1) In both control and extract-treated cultures incubated with monoclonal antibody $\mathrm{C} 1$, fluorescein-second antibody, and rhodaminebungarotoxin, 80 to $95 \%$ of the patches stained by either fluorophore were stained by both (see above). Sometimes, however, the correspondence was spatially imperfect; in Figure 2, for example, rhodamine- and fluoresceinstained areas do not coincide precisely. (2) Similarly, other antibodies to synaptic BL-JS1, JS2, JS3, and C4-usually stained AChR-rich regions. (3) In myotubes stained with bungarotoxin and an antibody to $\mathrm{AChE}$, nearly all AChE-rich patches were at AChR-rich sites. However, most bungarotoxin-stained areas did not bear 
TABLE I

Structures resembling junctional folds in cultured myotubes

Invaginations of the surface membrane that resembled junctional folds were counted on electron micrographs of randomly selected, crosssectioned myotubes. Invaginations were counted as folds if they were more than $0.1 \mu \mathrm{m}$ wide, deeper than wide, continuous with extracellular space, and coated with BL. See Figures $3 b, 7 b$, and $8 b$, for examples. Cultures were fixed 11 to 15 days after plating.

\begin{tabular}{|c|c|c|c|}
\hline & \multicolumn{3}{|c|}{ Type of Culture } \\
\hline & $\begin{array}{l}\text { Control } \\
\text { (Active) }\end{array}$ & $\begin{array}{c}\text { TTX- } \\
\text { treated } \\
\text { (Paralyzed) }\end{array}$ & $\begin{array}{c}\text { Brain } \\
\text { Extract- } \\
\text { treated }\end{array}$ \\
\hline No. of cultures & 20 & 5 & 18 \\
\hline No. of myotubes & 476 & 96 & 449 \\
\hline No. of folds ${ }^{a}$ & 7 & 4 & 123 \\
\hline Folds per myotube & 0.01 & 0.04 & 0.27 \\
\hline Percentage of myotubes with folds & $\begin{array}{c}1 \\
(4 / 476)\end{array}$ & $\begin{array}{c}4 \\
(4 / 96)\end{array}$ & $\begin{array}{c}15 \\
(67 / 449)^{b}\end{array}$ \\
\hline
\end{tabular}

${ }^{a}$ If invaginations were not required to be BL-coated to be counted as folds, totals were 8,5 , and 125 for control, paralyzed, and extracttreated myotubes; that is, almost all fold-like invaginations are BLcoated.

${ }^{b}$ Differs from control and paralyzed cultures at $p<0.001$ by $\chi^{2}$ test.
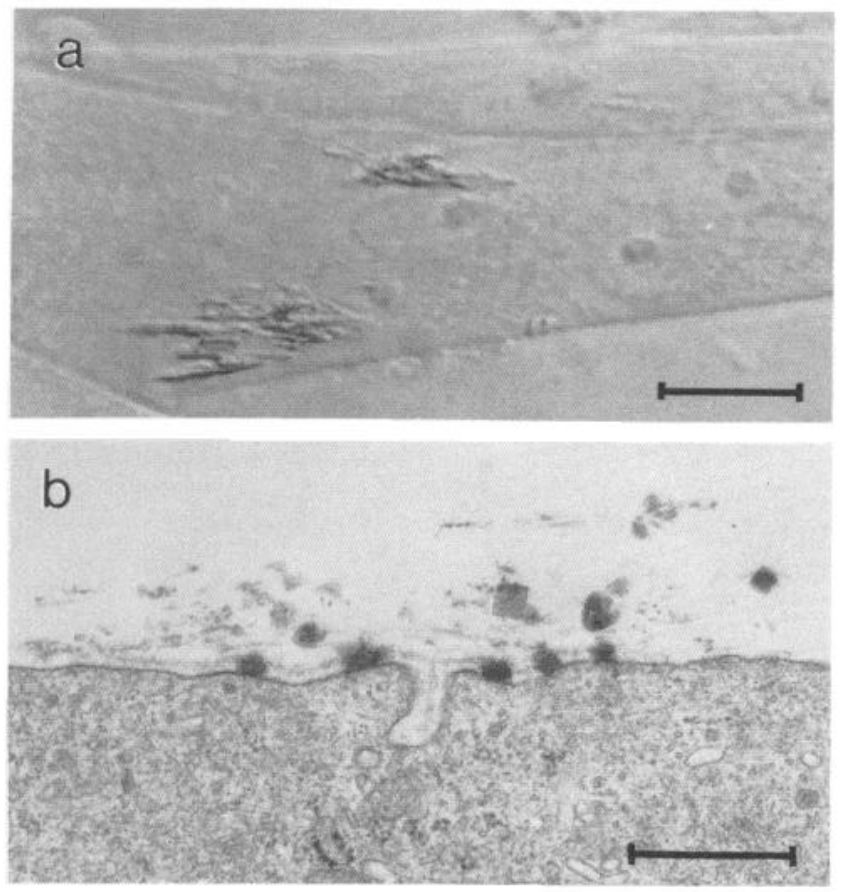

Figure 8. AChE-rich patches on the surface of histochemically stained, extract-treated myotubes. $a$, Light micrograph of two patches, photographed with Nomarski optics. $b$, Electron micrograph of an AChE-rich region, marked by granular reaction product, that is coated with $\mathrm{BL}$ and bears a fold. The bar is $20 \mu \mathrm{m}$ in $a$ and $0.5 \mu \mathrm{m}$ in $b$.

immunocytochemically detectable AChE, and AChRrich patches outnumbered histochemically stained AChE-rich patches by a ratio of 5 to $8: 1$ in both control and extract-treated cultures (Fig. 5). (4) Electron microscopy showed that folds occurred largely if not entirely in association with other synaptic specializations. Reaction product covered and lined all of 54 folds found in myotubes that had been stained with antisynaptic BL and HRP-second antibody, all of 10 folds found in HRPbungarotoxin-stained myotubes, and 36 of 37 folds found in AChE-stained myotubes. Most AChR and synaptic BL-rich regions viewed in cross-sections were not enfolded, but in the absence of serial sections one does not know whether a fold was nearby. However, as noted above, the majority of AChE-stained sites were associated with folds. Our results are all consistent with the idea that synaptic BL antigens and AChRs are almost always clustered together in small patches, a subset of which contain $\mathrm{AChE}$ and junctional folds.

\section{Discussion}

One way in which a nerve might influence the differentiation of its target is by releasing a soluble factor that could affect the organization or metabolism of the postsynaptic cell. If such a factor could act locally, it could account in part for the restricted localization of molecules and structures to synaptic sites. In investigating this possibility, several workers have shown that addition of neural extracts to muscle cells cultured without neurons can induce the formation of AChR-rich patches in the myotube membrane (Christian et al., 1978; Podleski et al., 1978; Jessell et al., 1979; Kalcheim et al., 1982a, b; Markelonis et al., 1982; Salpeter et al., 1982). We have found that an extract of adult rat brain can also induce the formation of patches of BL that resemble synaptic but not extrasynaptic BL of adult muscle. They contain high concentrations of several synaptic BL antigens, focal accumulations of $\mathrm{AChE}$, and projections that extend into folds in the myotube surface. Double-label experiments show that these features usually occur together and overlie AChR-rich patches of plasma membrane. Extract causes a large increase in the fraction of the myotube surface covered by synaptic BL without a comparable change in the overall fraction of the surface covered by BL. Thus, brain extract induces synapse-like differentiation of $\mathrm{BL}$ as it does of plasma membrane.

Our results are consistent with recent reports that clusters of AChRs induced by neural extracts are covered by BL (Daniels et al., 1981; Salpeter et al., 1982) and that neural extracts can stimulate the synthesis of AChE (Jessell et al., 1979; Markelonis et al., 1982) and collagen (Kalcheim et al., 1982a, b) in muscle cultures. Junctional folds and focal accumulations of AChE have not previously been reported to occur in rat myotubes cultured without nerve (Rieger et al., 1980; Salpeter et al., 1982), although both of these features have been detected in rat nerve-muscle cultures (Koenig and Vigny, 1978; Nakajima et al., 1980) and in aneural cultures of Xenopus muscle (Moody-Corbett and Cohen, 1981; Weldon et al., 1981; Peng and Cheng, 1982). Focal accumulations of AChE also appear on myotubes of a mouse myogenic cell line, where they have been shown to be associated with extracellular matrix, presumably BL (Inestrosa et al., 1982); this result,along with our observation that $\mathrm{BL}$ is almost invariably present in enzyme-rich areas, supports the presumption that $\mathrm{AChE}$ in patches is attached to BL.

The spatial correspondence of several specialized features of $\mathrm{BL}$ and their coordinate induction by brain 
extract raise questions about the way in which the extract works. For example, one wants to know whether the same components of the extract are responsible for the observed effects on AChRs and BL and for the several effects on BL. Recent progress in purification of receptor-clustering factors (e.g., Bauer et al., 1981; Markelonis et al., 1982; Buc-Caron et al., 1983) may soon allow resolution of this question. It will also be important to learn the mechanisms by which brain extract exerts its effects on BL. Extract might affect the synthesis, degradation, distribution, and/or assembly of BL components, or even directly contribute some components to the BL. Some effects of the extract on BL may be indirect. For example, concentration of synaptic BL antigens in high-density patches could induce folding of the membrane or provide binding sites for AChE. It is also possible that clustering of AChRs and of BL antigens is causally related (see Sanes, 1983). The availability of monoclonal antibodies to components of BL (Anderson and Fambrough, 1982; Sanes and Chiu, 1983) will make it possible for us to study these molecular questions.

The regulatory mechanisms we have demonstrated in vitro (summarized in Fig. 9) suggest ways in which nerve could influence accumulation of myotube $\mathrm{BL}$ in vivo. Sanes and Lawrence (1983) found that spontaneously active myotubes accumulate more total BL but fewer synaptic BL-rich patches than chronically paralyzed myotubes (Fig. 9, $a$ and $b$ ). We showed here that extract-

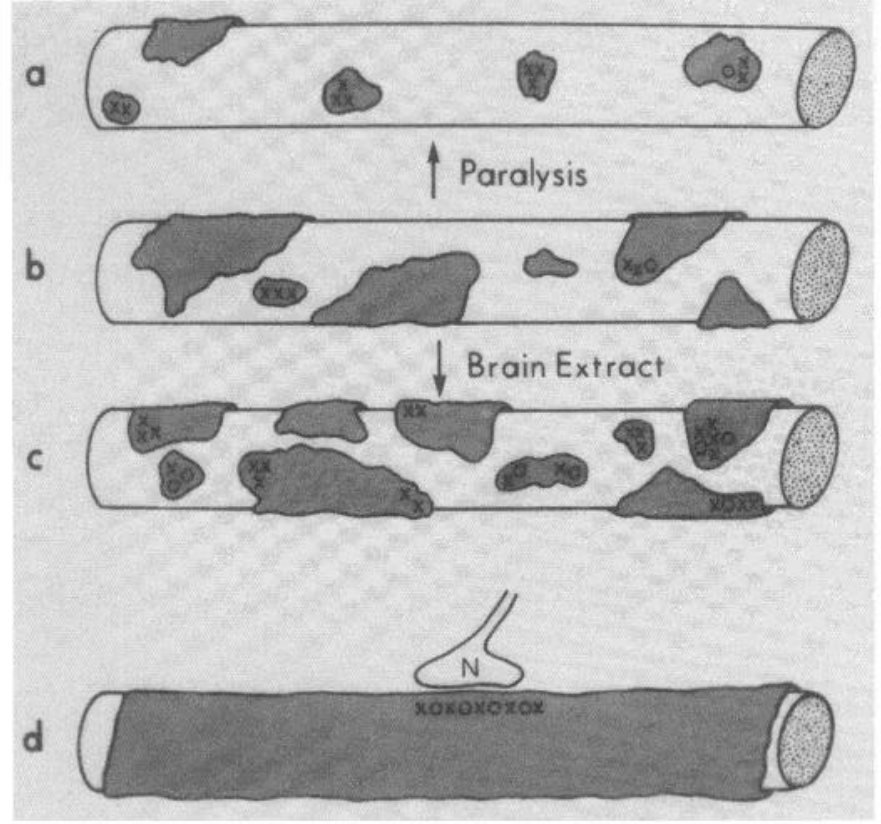

Figure 9. The BL of cultured myotubes: schematic summary of results obtained from immunocytochemical, histochemical, and conventional staining of chronically paralyzed $(a)$, control $(b)$, and brain extract-treated $(c)$ cultures. $d$ shows an innervated adult muscle fiber. $N$, nerve terminal. Shaded areas are BL-covered. $x$, synaptic $\mathrm{BL}$ antigen-rich patches; $o$, regions with folds and/or concentrations of AChE. Paralyzed myotubes accumulate less total BL and AChE but more patches rich in synaptic antigens than controls. Extract-treated myotubes accumulate many more synaptic antigen-, AChE-, and fold-rich patches than controls with little change in total BL. treated myotubes differ little from controls in total $\mathrm{BL}$ but assemble far more synaptic BL-rich regions, some of which bear junctional folds and AChE (Fig. 9, $b$ and $c$ ). In the embryo, a motor axon could synaptically evoke electrical or contractile activity and thus induce immature, BL-poor myotubes to accumulate BL. By releasing some soluble agent(s), the nerve might induce or stabilize accumulation of synaptic BL, junctional folds, and $\mathrm{AChE}$ in its vicinity. Activity would prevent or reverse the formation of synaptic antigen-rich BL everywhere except at the synapse. The result would be a muscle uniformly coated with BL but with synaptic BL antigens, AChE, and junctional folds concentrated at synaptic sites (Fig. $9 d)$. This hypothesis is almost certain to be incomplete, since AChRs, AChE, synaptic BL antigens, and junctional folds each accumulate at a different time during synapse formation in vivo (e.g., Kelly and Zacks, 1969; Chiu and Sanes, 1982; Weinberg et al., 1981). Nevertheless, the activity-dependent and -independent mechanisms that we have found in myotubes cultured without neurons could be involved in mediating nerve-muscle interactions during synaptogenesis, and direct tests of our hypothesis should be feasible in nerve-muscle cocultures and in vivo.

\section{References}

Anderson, M. J., and D. M. Fambrough (1982) Nerve-induced deposition of basal lamina proteoheparan sulphate during development of the neuromuscular junction. J Cell Biol. 95: $120 \mathrm{a}$.

Bauer, H.C., M. P. Daniels, P.A. Pudimat, L. Jacques, H. Sugiyama, and C. N. Christian (1981) Characterization and partial purification of a neuronal factor which increases acetylcholine receptor aggregation on cultured muscle cells. Brain Res. 209: 395-404.

Brockman, S. K., R. J.Przybylski, and S. G. Younkin (1982) Cellular localization of the molecular form of acetylcholinesterase in cultured embryonic rat myotubes. J. Neurosci. 2: 1775-1785.

Buc-Caron, M., P. Nystrom, and G. D. Fischbach (1983) Induction of acetylcholine receptor synthesis and aggregation partial purification of low-molecular-weight activity. Dev. Biol. 95: 378-386.

Cangiano, A., T. Lømo, L. Lutzemberger, and O. Sveen (1980) Effects of chronic nerve conduction block on formation of neuromuscular junctions and junctional acetylcholinesterase in the rat. Acta Physiol.Scand. 109: 283-296.

Chiu, A. Y., and J. R. Sanes (1982) Differentiation of basal lamina is an early event in the development of neuromuscular junctions in vivo. Soc. Neurosci. Abstr. 8: 128.

Christian, C. N., M. P. Daniels, H. Sugiyama, Z. Vogel, L. Jacques, and P. G. Nelson (1978) A factor from neurons increases the number of acetylcholine receptor aggregates on cultured muscle cells. Proc. Natl. Acad. Sci. U. S. A. 75: 40114015.

Chung, A. E., R. Jaffe, I. L. Freeman, J. -P. Vergnes, J. E. Braginski, and B. Carlin (1979) Properties of a basement membrane-related glycoprotein synthesized in culture by a mouse embryonal carcinoma-derived cell line. Cell 16: 277287.

Cohen, S. A., nd G. D. Fischbach (1973) Regulation of muscle acetylcholine sensitivity by muscle activity in cell culture. Science 181: 76-78.

Daniels, M. P., M. Vigny, H. Bauer, P. Sonderegger, and Z.Vogel (1981) Laminin and other basement membrane proteins codistribute with acetylcholine receptors on the surface 
of cultured myotubes. Soc. Neurosci.Abstr. 7: 143.

Ellman, G. L., K.D. Courtney, V. Andres, and R. M. Featherstone (1961) A new and rapid colorimetric determination of acetylcholinesterase activity. Biochem. Pharmacol. 7: 88-95.

Greenberg, A. J., K. K. Parker, and A. J. Trevor (1977) Immunochemical studies of mammalian brain acetylcholinesterase. J. Neurochem. 29: 911-917.

Harris, A. J. (1980) Trophic effects of nerve on muscle. The Physiology of Peripheral Nerve Disease, A. J. Sumner, ed., pp. 195-220, W. B. Saunders, Philadelphia.

Inestrosa, N. C., L. Silberstein, and Z. W. Hall (1982) Association of the synaptic form of acetylcholinesterase with extracellular matrix in cultured mouse muscle cells. Cell 29: 7179 .

Jessell, T. M., R. E. Siegel, and G.D. Fischbach (1979) Induction of acetylcholine receptors on cultured skeletal muscle by a factor extracted from brain and spinal cord. Proc. Natl. Acad. Sci. U. S. A. 76: 5397-5401.

Kalcheim, C., D. Duksin, and Z. Vogel (1982a) Involvement of collagen in the aggregation of acetylcholine receptors on cultured muscle cells. J. Biol. Chem. 257: 12722-12727.

Kalcheim, C., Z. Vogel, and D. Duksin (1982b) Embryonic brain extract induces collagen biosynthesis in cultured muscle cells: Involvement in acetylcholine receptor aggregation. Proc. Natl. Acad. Sci. U. S. A. 79: 3077-3081.

Karnovsky, M. J. (1964) The localization of cholinesterase activity in rat cardiac muscle by electron microscopy. J. Cell Biol. 23: 217-232.

Kelly, A. M., and S. I. Zacks (1969) The fine structure of motor endplate morphogenesis. J. Cell Biol. 42: 154-169.

Koelle, G. B. (1963) Cholinesterases and Anticholinesterase Agents. Handbuch der experimentellen pharmakologie, XV, Springer-Verlag, Berlin.

Koenig, J., and M. Vigney(1978) Neural induction of the $16 \mathrm{~S}$ acetylcholinesterase in muscle cell cultures. Nature 271: 7577.

Kuhl, V., R. Timpl, and K. Von der Mark (1982) Synthesis of Type IV collagen and laminin in cultures of skeletal muscle cells and their assembly on the surface of myotubes. Dev. Biol. 93: 344-354.

Lawrence, J. C., and W. A. Catterall (1981) Tetrodotoxininsensitive sodium channels. Binding of polypeptide neurotoxins in primary cultures of rat muscle cells. J. Biol. Chem. 256: 6223-6229.

Lentz, T. L., J. E. Mazurkiewicz, and J. Rosenthal (1977) Cytochemical localization of acetylcholine receptors at the neuromuscular junction by means of horseradish peroxidaselabeled $\alpha$-bungarotoxin. Brain Res. 132: 423-442.

Lømo, T., and C. R. Slater (1980) Control of junctional acetylcholinesterase by neural and muscular influences in the rat. J. Physiol. (Lond.) 303: 191-202.

Markelonis, G. J., T. H. Oh, M. E. Eldefrawi, and L. Guth (1982) Sciatin: A myotrophic protein increases the number of acetylcholine receptors and receptor clusters in cultured skeletal muscle. Dev. Biol. 89: 353-361.

Massoulie, J., and S. Bon (1982) The molecular forms of cholinesterase and acetylcholinesterase in vertebrates. Annu. Rev. Neurosci. 5: 57-106.

Moody-Corbett, F., and M. W. Cohen (1981) Localization of cholinesterase at sites of high acetylcholine receptor density on embryonic amphibian muscle cells cultured without nerve. J. Neurosci. 1: 596-605.

Nakajima, Y., Y. Kidokoro, and F. G. Klier (1980) The development of functional neuromuscular junctions in vitro: An ultrastructural and physiological study. Dev. Biol. 77: 52-72.

Novikoff, A. B., P. M. Novikoff, N. Quintana, and C. Davis (1972) Diffusion artifacts in 3, 3-diaminobenzidine cytochemistry. J. Histochem. Cytochem. 20: 745-749.

Oh, T. H. (1975) Neurotrophic effects: Characterization of the nerve extract that stimulates muscle development in culture. Exp. Neurol. 46: 432-438.

Peng, H. B., and P. C. Cheng (1982) Formation of postsynaptic specializations induced by latex beads in cultured muscle cells. J. Neurosci. 2: 1760-1774.

Podleski, T. R., D. Axelrod, P. Ravdin, I. Greenberg, M. M. Johnson, and M. M. Salpeter (1978) Nerve extract induces increases and redistribution of acetylcholine receptors on cloned muscle cells. Proc. Natl. Acad. Sci. U. S. A. 75: 20352039.

Purves, D. (1976) Long term regulation in the vertebrate peripheral nervous system. In International Review of Physiology, Neurophysiology II, R. Porter, ed., pp. 125-177, University Park Press, Baltimore.

Rieger, F., J. Koenig, and M. Vigny (1980) Spontaneous contractile activity and the presence of the $16 \mathrm{~S}$ form of acetylcholinesterase in rat muscle cells in culture: Reversible suppressive action of tetrodotoxin. Dev. Biol. 76: 358-365.

Rubin, L. L., S. M. Schuetze, C. L. Weill, and G. D. Fischbach (1980) Regulaton of acetylcholinesterase appearance at neuromuscular junctions in vitro. Nature 283: 264-267.

Sakashita, S., E. Engvall, and E. Ruoslahti (1980) Basement membrane glycoprotein laminin binds to heparin. FEBS Lett. 116: 243-246.

Salpeter, M. M., S. Spanton, K. Holley, and T. R. Podleski (1982) Brain extract causes acetylcholine receptor redistribution which mimics some early events at developing neuromuscular junctions. J. Cell Biol. 93: 417-425.

Sanes, J. R. (1982) Laminin, fibronectin, and collagen in synaptic and extrasynaptic portions of muscle fiber basement membrane. J. Cell Biol. 93: 442-451.

Sanes, J. R. (1983) Roles of extracellular matrix in neural development. Annu. Rev. Physiol. 45: 581-600.

Sanes, J. R., and A. Y. Chiu (1983) The basal lamina of the neuromuscular junction. Cold Spring Harbor Symp. Quant. Biol. 48: 667-678.

Sanes, J.R., and Z. W. Hall (1979) Antibodies that bind specifically to synaptic sites on muscle fiber basal lamina. J. Cell Biol. 83: 357-370.

Sanes, J. R., and J. C. Lawrence, Jr. (1983) Activity-dependent accumulation of basal lamina by cultured rat myotubes. Dev. Biol. 97: 123-136.

Sanes, J. R., L. M. Marshall, and U. J. McMahan (1978) Reinnervation of muscle fiber basal lamina after removal of myofibers. Differentiation of regenerating axons at original synaptic sites. J.Cell Biol. 78: 176-198.

Sanes, J. R., D. H. Feldman, and J. C. Lawrence, Jr. (1982) Activity and neural extract regulate accumulation of basal lamina by cultured myotubes. Soc. Neurosci. Abstr. 8: 129.

Silberstein, L., N. Inestrosa, and Z. W. Hall (1982) Aneural muscle cell cultures make synaptic basal lamina components. Nature 295: 143-145.

Streit, P., and J. C. Reubi (1977) A new and sensitive staining method for axonally transported horseradish peroxidase (HRP) in the pigeon visual system. Brain Res. 126: 530-537.

Strohman, R. C., E. Bandman, and C. R. Walker (1981). Regulation of myosin accumulation by muscle activity in cell culture. J. Muscle Res. Cell Motil. 2: 269-282.

Weinberg, C. B., J. R. Sanes, and Z. W. Hall (1981) Formation of neuromuscular junctions in adult rats: Accumulation of acetylcholine receptors, acetylcholinesterase, and components of synaptic basal lamina. Dev. Biol. 84: 255-266.

Weldon, P. R., F. Moody-Corbett, and M. W. Cohen (1981) Ultrastructure of sites of cholinesterase activity on amphibian embryonic muscle cells cultured without nerve. Dev. Biol. 84: $341-350$.

Zacks, S. I., M. R. Sheff, and A. Saito (1973) Structure and staining characteristics of myofiber external lamina. J. Histochem. Cytochem. 21: 703-714. 